\title{
Nonlinear Landing Control for Quadrotor UAVs
}

\author{
Holger Voos \\ University of Applied Sciences Ravensburg-Weingarten, Mobile Robotics Lab, \\ D-88241 Weingarten
}

\begin{abstract}
Quadrotor UAVs are one of the most preferred type of small unmanned aerial vehicles because of the very simple mechanical construction and propulsion principle. However, the nonlinear dynamic behavior requires a more advanced stabilizing control and guidance of these vehicles. In addition, the small payload reduces the amount of batteries that can be carried and thus also limits the operating range of the UAV. One possible solution for a range extension is the application of a mobile base station for recharging purpose even during operation. However, landing on a moving base station requires autonomous tracking and landing control of the UAV. In this paper, a nonlinear autopilot for quadrotor UAVs is extended with a tracking and landing controller to fulfill the required task.
\end{abstract}

\section{Introduction}

Unmanned aerial vehicles (UAVs) already have a wide area of possible applications. Recent results in miniaturization, mechatronics and microelectronics also offer an enormous potential for small and inexpensive UAVs for commercial use. One very promising vehicle with respect to size, weight, construction and maneuverability is the so called quadrotor which is a system with four propellers in a cross configuration, see Fig. 1. By varying the speed of the single motors, the lift force can be changed and vertical and/or lateral motion can be generated. However, the quadrotor is a dynamically unstable nonlinear system which requires a suitable vehicle control system. One main drawback of small UAVs in nearly all types of application is the reduced payload and the limited amount of batteries that can be carried. Therefore the UAV has to return to a base station after a comparatively short amount of time for recharging purpose. In addition, in missions where a longer operating range is required such as pipeline, border or coast surveillance, returning to a stationary base station is also not useful. Instead it would be more suitable to operate with an autonomous mobile base station, e.g. a mobile robot or a ship, that is able to carry a higher amount of energy for several recharging cycles. Coordinated parallel operation of the mobile base station and the UAV then leads to an overall system for aerial surveillance with extended range. However, that concept requires basic stabilizing control of the quadrotor, tracking of the mobile base station and finally control of the landing procedure.

Concerning vehicle control design for small quadrotor UAVs, some solutions are already proposed in the literature, see e.g. [1-3] to mention only a few. Many 


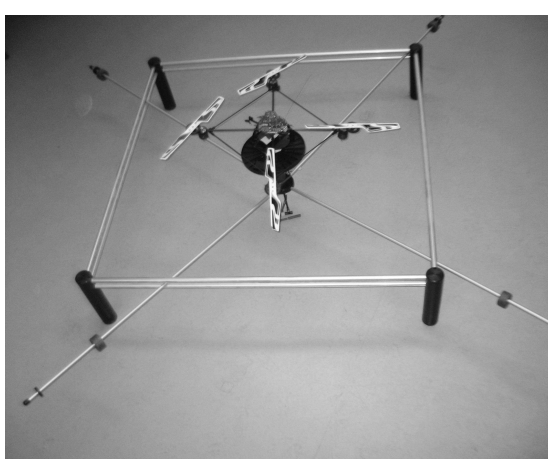

Fig. 1. A quadrotor in an experimental testbed.

of the proposed control systems are based on a linearized model and conventional PID- or state space control while other approaches apply sliding-mode, $\mathrm{H}_{\infty}$ or SDRE control. Recently, a new nonlinear control algorithm has been proposed by the author which is based upon a decomposition of the overall controller into a nested structure of velocity and attitude control. The controller has the advantage of an easy implementation and proven stability while taking the nonlinearities of the dynamics directly into account, see $[4,5]$ for further details. Here, this controller is used to provide the basis for the development of tracking and landing controller. Tracking and landing control has also been investigated in the literatur, see e.g. [6,7], while this paper proposes a new nonlinear approach which again has the advantage of an easy implementation and proven stability. These control strategies are eplained in some details here, first simulation and experimental results underline the obtained performance.

\section{The Vehicle Control System}

As previously described, landing control is based upon a vehicle control system which is applied to stabilize any desired velocity vector of the quadrotor. The overall control system as described in $[4,5]$ consist of an inner attitude and an outer velocity control loop. The command to the vehicle control system is a desired velocity vector in an inertial coordinate system given by $\boldsymbol{v}_{d}^{T}=\left(v_{x d}, v_{y d}, v_{z d}\right)$. For the attitude control, feedback linearization is applied that transforms the system into a decoupled linear system in state variable format. This resulting system is controlled via a conventional linear controller, leading to a sufficiently fast and damped closed loop dynamics. The outer control loop applies a nonlinear inversion to compensate a static nonlinearity and a linear control system to control the remaining linear dynamics. The advantage of the derived control system besides the excellent performance is the simple structure and the easy implementation. That leads to a fast computation even on standard embedded micro-controller systems. 
In order to evaluate the vehicle control system, an experimental prototype of the quadrotor has been designed (see Fig. 1), the dynamic model has been derived by identification of the system parameters like inertias, dimensions etc., and simulation results of vehicle control system have been obtained, see also $[4,5]$ for a more detailed description. Here, some results of the vehicle controller during experimental test flights are presented. In the experiment the control goal comprises the stabilization of the hovering state, i.e. $\boldsymbol{v}_{d}=\mathbf{0}$ and $\boldsymbol{\Omega}_{d}=\mathbf{0}$ (i.e. the Euler angles should be zero), starting from any initial deviations and compensating for any external disturbances. The obtained control result is shown in Fig. 2 as a time plot of all angles of the quadrotor. After a very short transition phase the hovering state is reached and maintained. The small constant deviation of the yaw angle $\psi$ results from a slight misalignment of the inertial measurement unit. It becomes obvious from Fig. 2 that external disturbances at $t=35 \mathrm{sec}$ of the roll angle, at $t=45 \mathrm{sec}$ of the pitch angle and at $t=50 \mathrm{sec}$ at the yaw angle are completely compensated.

The simulation and experimental results underline the performance of the developed vehicle controller, which is now used to stabilize the desired velocity vector commanded by the landing control system. However, the desig of a tracking and landing controller requires a dynamic model of the controlled quadrotor, i.e. the closed loop of quadrotor dynamics and vehicle controller. If we assume that the inner attitude control loops are sufficiently fast as designed in [5], the overall vehicle control system can be decomposed into three independent velocity control loops which can be approximated by linear first-order system, respectively, see e.g. the closed-loop dynamics with respect to $v_{x}$ :

$$
\frac{V_{x}(s)}{V_{x d}(s)} \approx \frac{1}{T_{x} \cdot s+1}
$$

with $T_{x}$ as appropriate time constant. A simulation of the step response also supports this approximation, see Fig. 3. Similar results are obtained for the step response of the two other velocities $v_{y}, v_{z}$. These first-order approximations of
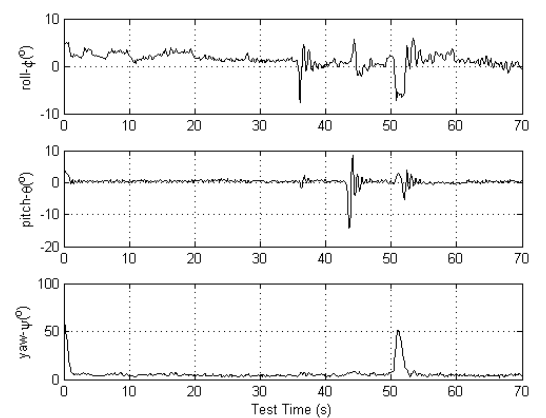

Fig. 2. Experimental results of the vehicle control system.

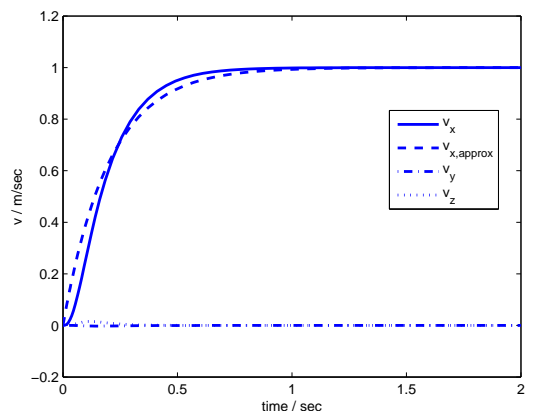

Fig. 3. Step response with regard to $v_{x}$. 
the controlled quadrotor UAV are now used for the design of the tracking and landing controllers.

\section{Automatic Landing on a Mobile Platform}

In the following we consider the problem that a quadrotor UAV stabilized via the previously described vehicle control system should land on a moving platform. The platform is moving on the surface of the underlying terrain at an altitude of $z_{s}(t)$ with regard to the inertial frame. The overall tracking and landing procedure can be decomposed into two independent control tasks: a tracking procedure in a pure $\mathrm{x}$-y-plane and an altitude control problem in pure z-direction. In the pure $x-y$-plane, only the planar mappings of the center of mass of the quadrotor and the platform and their respective motions are considered. The 2D-tracking controller has the task to reduce the planar distance between the quadrotor and the platform in this two-dimensional plane to zero and to maintain the zero distance even if disturbances occur. In parallel, an altitude controller has the task to achieve and stabilize required setpoints of the quadrotor's altitude over ground. During a first approaching phase where the planar distance between the quadrotor and the platform is above a threshold, the setpoint of the altitude over ground is set to a safety value, e.g. $5 \mathrm{~m}$. If the planar distance decreases below the defined threshold, e.g. $0.5 \mathrm{~m}$, the final landing procedure starts and the required setpoint of the altitude over ground is set to zero.

The result of the altitude controller is a desired velocity component in zdirection, i.e. $v_{z d}$ for the underlying vehicle controller while the result of the 2D-tracking controller are the two components of the desired velocity vector in $\mathrm{x}$ - and y-direction, i.e. $v_{x d}, v_{y d}$. Finally, the results of these two controllers form the overall desired velocity vector which is commanded to the vehicle controller. In the following, first the altitude controller is derived, then we discuss the developed 2D-tracking controller.

\subsection{Altitude Control}

The general task of the altitude control system is to achieve and maintain a desired altitude reference which can be either the constant altitude over ground during the approaching phase or a zero altitude over ground during the final landing phase. If $z$ is the altitude of the quadrotor UAV and $z_{s}$ is the current altitude of the surface (i.e. the platform) in the inertial frame, the difference $\Delta z=z-z_{s}$ is the relative altitude of the UAV over ground. The current desired altitude over ground commanded by the overall landing control is the value $\Delta z_{d}$. Now we assume that the dynamic behavior of the controlled quadrotor UAV in $z$-direction can be approximated by a first-order system (1), i.e.

$$
F_{Q}(s)=\frac{V_{z}(s)}{V_{z d}(s)} \approx \frac{1}{T_{z} \cdot s+1}
$$

If a linear altitude controller with transfer function $F_{R, z}(s)$ is chosen, the structure of the resulting closed altitude control loop can be depicted as shown in 
Fig. 4. Herein the altitude of the surface $z_{s}$ is considered as a non-measurable disturbance, however the quadrotor is able to measure the current altitude over ground $\Delta z$ with a suitable sensor system. In the literature some solutions based on ultrasonic, optical or laser sensors have already been proposed for this measurement problem, see e.g. [6,7]. It becomes obvious from the structure of the altitude control loop shown in Fig. 4 that a PD-controller can be applied in order to solve the control task:

$$
F_{R, z}(s)=K \cdot\left(1+T_{C} \cdot s\right)
$$

The parameters of the controller are adjusted in a way that the closed loop has zero overshoot (in order to avoid collisions with the platform during landing) and is sufficiently fast. The reference altitude $\Delta z_{d}$ is set to the desired safety altitude in the approach phase and set to zero in the landing phase.

\section{$3.2 \quad$ Nonlinear 2D-Tracking Controller}

The main goal of the tracking controller is to minimize the distance to a moving platform and to track this platform in the pure $\mathrm{x}-\mathrm{y}$-plane. For that purpose we consider a platform that is moving with the two velocity components $v_{P x}$ and $v_{P y}$ in $\mathrm{x}$ - and y-direction, respectively. The quadrotor is moving with the two velocity components $v_{Q x}$ and $v_{Q y}$, where the dynamics between the desired velocities $v_{Q x d}, v_{Q y d}$ and the actual velocities is given by a first-order system according to (1). The engagement geometry is depicted in Fig. 5, where $\sigma$ is the line-of-sight angle and $R$ is the distance or range between the quadrotor and the moving platform. It can be derived from classical missile guidance problems, see e.g. [8], that the relative kinematics can be described by the two differential

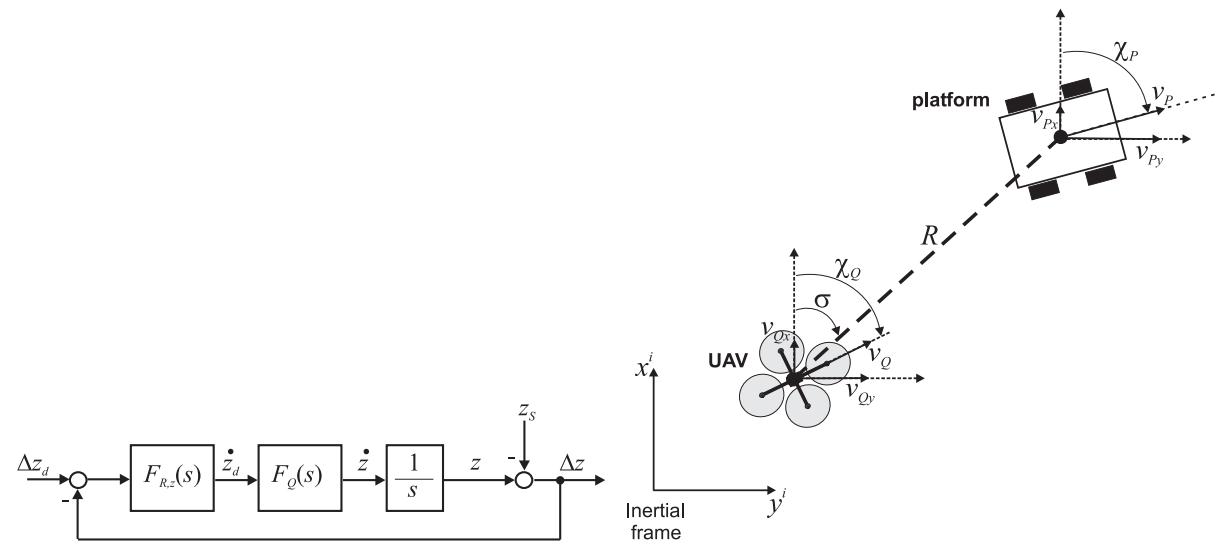

Fig. 4. Altitude control loop.

Fig. 5. Engagement geometry of quadrotor and mobile platform. 
equations:

$$
\begin{aligned}
\dot{R} & =v_{P x} \cos \sigma+v_{P y} \sin \sigma-v_{Q x} \cos \sigma-v_{Q y} \sin \sigma \\
\dot{\sigma} & =\frac{1}{R}\left(v_{P y} \cos \sigma-v_{P x} \sin \sigma-v_{Q y} \cos \sigma+v_{Q x} \sin \sigma\right)
\end{aligned}
$$

If we now again define a state variable model with the four state variables $x_{1}=$ $R, x_{2}=\sigma, x_{3}=v_{Q x}, x_{4}=v_{Q y}$, the input variables $u_{1}=v_{Q x d}, u_{2}=v_{Q y d}$ and the two measurable disturbance variables $d_{1}=v_{P x}, d_{2}=v_{P y}$, we finally obtain from (1), (4):

$$
\begin{aligned}
& \dot{x}_{1}=-x_{3} \cos x_{2}-x_{4} \sin x_{2}+d_{1} \cos x_{2}+d_{2} \sin x_{2} \\
& \dot{x}_{2}=\frac{1}{x_{1}}\left(x_{3} \sin x_{2}-x_{4} \cos x_{2}-d_{1} \sin x_{2}+d_{2} \cos x_{2}\right) \\
& \dot{x}_{3}=-\left(1 / T_{1}\right) \cdot x_{3}+\left(1 / T_{1}\right) \cdot u_{1} \\
& \dot{x}_{4}=-\left(1 / T_{2}\right) \cdot x_{4}+\left(1 / T_{2}\right) \cdot u_{2}
\end{aligned}
$$

For the design of a suitable controller we first consider a suitable operating point. This is the state where the range and line-of-sight angle are zero and the quadrotor moves in accordance with the platform, i.e. $x_{1}=0, x_{2}=0, x_{3}=$ $d_{1}, x_{4}=d_{2}$. We define the Lyapunov function $V\left(x_{1}, x_{2}, x_{3}, x_{4}\right)$ which is $C^{1}$ and positive defined around the operating point:

$$
V\left(x_{1}, x_{2}, x_{3}, x_{4}\right)=0.5 \cdot\left(x_{1}^{2}+x_{2}^{2}+\left(x_{3}-d_{1}\right)^{2}+\left(x_{4}-d_{2}\right)^{2}\right)
$$

Now we calculate the first derivative of $V$ using (5) and assume that the platform moves with a constant velocity:

$$
\begin{aligned}
\dot{V} & =x_{1} \dot{x}_{1}+x_{2} \dot{x}_{2}+\left(x_{3}-d_{1}\right) \dot{x}_{3}+\left(x_{4}-d_{2}\right) \dot{x}_{4} \\
& =-\frac{x_{3}^{2}}{T_{1}}-\frac{x_{4}^{2}}{T_{2}}+x_{3} f_{1}+x_{4} f_{2}+d_{1} f_{3}+d_{2} f_{4}
\end{aligned}
$$

with

$$
\begin{aligned}
& f_{1}=-x_{1} \cos x_{2}+\frac{x_{2}}{x_{1}} \sin x_{2}+\frac{1}{T_{1}} u_{1}+\frac{1}{T_{1}} d_{1} \\
& f_{2}=-x_{1} \sin x_{2}-\frac{x_{2}}{x_{1}} \cos x_{2}+\frac{1}{T_{2}} u_{2}+\frac{1}{T_{2}} d_{2} \\
& f_{3}=x_{1} \cos x_{2}-\frac{x_{2}}{x_{1}} \sin x_{2}-\frac{1}{T_{1}} u_{1} \\
& f_{4}=x_{1} \sin x_{2}+\frac{x_{2}}{x_{1}} \cos x_{2}-\frac{1}{T_{2}} u_{2}
\end{aligned}
$$

This derivative must be negative defined in order to guarantee that the operating point is asymptotically stable. Using $(7),(8)$ we first set $f_{3}=-\left(1 / T_{1}\right) \cdot d_{1}$ and $f_{4}=-\left(1 / T_{2}\right) \cdot d_{2}$ which yields

$$
\begin{aligned}
& u_{1}=d_{1}+T_{1} x_{1} \cos x_{2}-T_{1} \frac{x_{2}}{x_{1}} \sin x_{2} \\
& u_{2}=d_{2}+T_{2} x_{1} \sin x_{2}+T_{2} \frac{x_{2}}{x_{1}} \cos x_{2}
\end{aligned}
$$


Inserting this in $f_{1}, f_{2}$ using (8) leads to $f_{1}=\left(2 / T_{1}\right) \cdot d_{1}$ and $f_{2}=\left(2 / T_{2}\right) \cdot d_{2}$ which finally results in

$$
\dot{V}=-\frac{1}{T_{1}}\left(x_{3}-d_{1}\right)^{2}-\frac{1}{T_{2}}\left(x_{4}-d_{2}\right)^{2}
$$

This proofs that the derivative of $V$ is negative defined and the operating point is asymptotically stable if the tracking control law (9), (10) is applied. However, in order to obtain a limited control input, we set $u_{1}=d_{1}, u_{2}=d_{2}$ if the range $x_{1}$ becomes smaller than a defined very small threshold.

In order to apply this $2 \mathrm{D}$-tracking controller, the range $x_{1}=R$, the line-ofsight angle $x_{2}=\sigma$ as well as the velocity components $d_{1}=v_{P x}, d_{2}=v_{P y}$ of the platform must be measured. Both $R$ and $\sigma$ can be easily calculated if the positions of the quadrotor and the platform in the inertial frame are measured. In addition it is assumed that the platform also measures its velocity components. Both position and velocity components of the platform are transmitted via communication to the quadrotor, resulting in a cooperative approach. Regarding the measurements, a DGPS is applied for the determination of the positions, respectively, during the approach phase. However, more accurate measurements are necessary during the landing phase. There are some possible solutions for this problem such as a vision based or ultrasonic based sensor system, see e.g. $[6,7]$. The velocity components of the platform could be measured with a suitable inertial measurement unit onboard. Since the main focus of this work is on the development of the control system, we do not go into further details of the measurements but describe some first simulation results in the next chapter.

\section{Simulation and Experimental Results}

The overall landing control system is not yet implemented in the experimental quadrotor prototype and is therefore evaluated in simulations. The simulation consists of a nonlinear dynamic model of the quadrotor of order 12, the vehicle
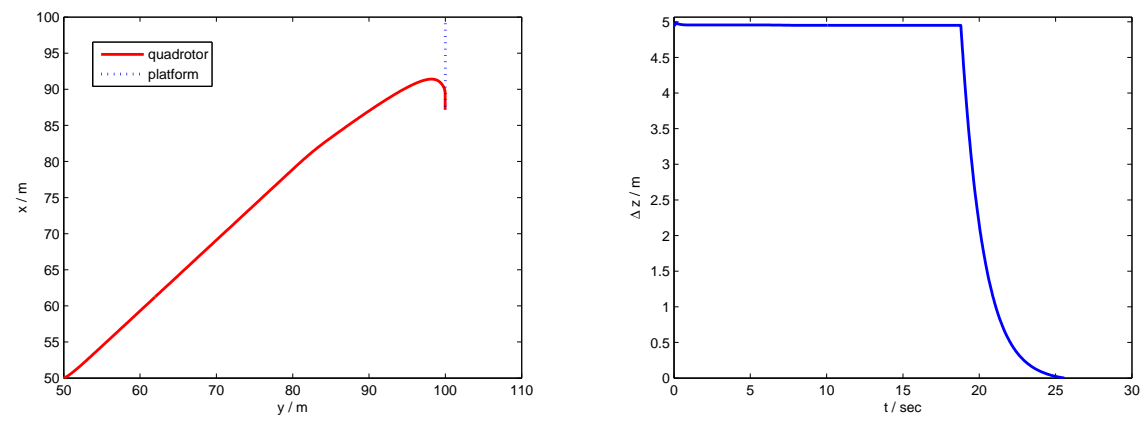

Fig. 6. Simulation of the overall landing control system. 
control system as described in [4,5] and the derived landing control system as well as a simulated moving platform. In the simulation, the platform is initially assumed to be located at $\left(x_{P 0}=100 \mathrm{~m}, y_{P 0}=100 \mathrm{~m}\right.$ and moving with a constant speed of $V_{P x}=-0.5 \mathrm{~m} / \mathrm{sec}$ in the x-y-plane. The quadrotor is initially located at $\left(x_{Q 0}=50 \mathrm{~m}, y_{Q 0}=50 \mathrm{~m}\right.$ in the inertial frame at an altitude of $\Delta z_{0}=5 \mathrm{~m}$. The obtained control result of the overall landing control system is depicted in Fig. 6. Diagram (a) shows a top view of the 2D-engagement in which the quadrotor starts from the initial position, tracks the path of the moving platform and finally lands on the platform. Diagram (b) shows the altitude of the quadrotor which descends from the initial altitude over ground until the final landing.

\section{Conclusion and Future Works}

This paper presents an overall control system for the automatic landing of a quadrotor UAV on a moving platform. Herein, the vehicle control system comprises a nonlinear inner loop attitude control and an outer loop velocity control system based on static inversion. The vehicle control system is finally realized in an experimental prototype and first test flights proof the performance of this novel nonlinear approach. The landing controller consists of a linear altitude controller and a nonlinear 2D-tracking controller. Simulation results finally underline the performance of the landing control system. In our ongoing work we are currently also implementing the landing control system as well as the necessary sensors in the UAV prototype.

\section{References}

1. Bouabdallah S, Siegwart R: Backstepping and Sliding-mode Techniques Applied to an Indoor Micro Quadrotor. IEEE Int Conf on Robotics and Automation, pp. 2247-2252, 2005.

2. Tayebi A, McGilvray S: Attitude Stabilization of a VTOL Quadrotor Aircraft. IEEE Trans on control systems technology, Vol. 14, pp. 562-571, 2006.

3. Castillo P, et al.: Real-time stabilization and tracking of a four-rotor mini rotorcraft. IEEE Trans on Control Systems Technology, VOL.12, No. 4, July 2004, pp. 510- 516.

4. Voos H: Nonlinear Control of a Quadrotor Micro-UAV using FeedbackLinearization. IEEE Int Conf on Mechatronics, Málaga, Spain, 2009.

5. Voos H: Entwurf eines Flugreglers fuer ein vierrotoriges unbemanntes Fluggeraet (in German). at-Automatisierungstechnik, 57(9), 2009. (to appear)

6. Waslander SL, et al.: Multi-agent quadrotor testbed control design: integral sliding mode vs. reinforcement learning. IEEE/RSJ International Conference on Intelligent Robots and Systems(IROS 2005), 2005.

7. Barber DB, et al.: Autonomous Landing of Miniature Aerial Vehicles. Brigham Young University Press, Provo, UT.

8. Zarchan P: Tactical and Strategic Missile Guidance. AIAA Press, 2007. 